\title{
Cryo-EM Analysis and de Novo Modeling of E. coli dihydrolipoamide Succinyltransferase Catalytic Domain Structure
}

\author{
Vasily Mikirtumov ${ }^{1}$, Evgeny Pichkur ${ }^{2}$, Olga Tikhonova ${ }^{3}$, Lidia Kurochkina ${ }^{4}$ and Olga Sokolova ${ }^{5}$ \\ ${ }^{1}$ Moscow Lomonosov University, moscow, Moskva, Russia, ${ }^{2}$ Kurchatov Institute, Moscow, Moskva, \\ Russia, ${ }^{3}$ Orekhovich Research Institute of Biomedical Chemistry RAS, Moscow, Moskva, Russia, \\ ${ }^{4}$ Belozersky Research Institute of Physico-Chemical Biology, Lomonosov Moscow State University, \\ Moscow, Moscow, Moskva, Russia, ${ }^{5}$ Lomonosov Moscow State University, Faculty of Biology, Moscow, \\ Moskva, Russia
}

Cryo-EM has recently been recognized as a major tool for obtaining protein structures at near-atomic resolution. The use of this technique has skyrocketed in recent years, due to certain technological advancements. This includes not only direct detectors and phase plates, but also software tools such as Relion [1], cryoSparc [2], and Coot [3]. At this stage, biochemical protein purification remains a bottleneck. This step is difficult to automate because it relies substantially on human expertise. A number of problems associated with the difficulties of choosing a protein expression system, method of purification, and concentration of functional protein remain. The overall goal is to minimize the number of purification steps, to minimize the impact of purification procedures on the object and to obtain a suitable amount of pure sample in a native environment.

Here, we produced a roughly purified cocktail of E.coli proteins using robust salt precipitation and solved the near-atomic resolution structure of an abundant $E$. coli protein, which was found in this cocktail by mass-spectrometry: the catalytic domain of dihydrolipoamide succinyltransferase (DSCD) without affinity tags (Fig. 1A, B). De novo structure modeling of DSCD in COOT produced an atomic map (Fig. $1 \mathrm{C})$ that revealed differences in positions of active center residues, in comparison to previously obtained crystal structures $[4,5]$. It allowed us to model the ligand binding and study in detail the conformation of the active site of the enzyme (Fig. 2A).

The comparison of our cryo-EM structure to the previous X-ray structure (Fig. 2B) demonstrated that there are some differences in side-chain orientations of key amino acids in the active center: Arg382, Arg185, and Asp380. The main yet unsolved question regarding the structure of DSCD - alterations of side chains orientation, which occur in the active site, due to substrate binding. It has been proposed that, upon Coenzyme A binding, Asp380 forms salt-bridges with His376 and either Arg 185 or Arg382 [4]. To form a salt bridge with one of the Arginines, Asp380 must change its orientation in a substrate-dependent manner. The cryo-EM density map allowed us to precisely place Arg185 and Arg382 side chains; these side chains have been disordered in the first X-ray study (1e2o) and identified in the most recent study (6prb). Comparison of models revealed that in our de novo model the Arginines are rotated. The distances between His376 and Arg in positions 382 and 185 were 4.87A and 5.77A, correspondingly. Thus, in our near-atomic cryo-structure, the active site possesses a slightly alternative conformation [6]. 
A

MSSVDILVPDLPESVADATVATWHKKPGDAVVRDEVLVEIETDKVVLEVPASADGILDAVLEDEGTTVTS

RQILGRLREGNSAGKETSAKSEEKASTPAQRQQASLEEQNNDALSPAIRRLLAEHNLDASAIKGTGVGGRL

TREDVEKHLAKAPAKESAPAAAAPAAQPALAARSEKRVPMTRLRKRVAERLLEAKNSTAMLTTFNEVNM

KPIMDLRKQYGEAFEKRHGIRLGFMSFYVKAVVEALKRYPEVNASIDGDDVVYHNYFDVSMAVSTPRGL

VTPVLRDVDTLGMADIEKKIKELAVKGRDGKLTVEDLTGGNFTITNGGVFGSLMSTPIINPPQSAILGMHAI

KDRPMAVNGQVEILPMMYLALSYDHRLIDGRESVGFLVTIKELLEDPTRLLLDV
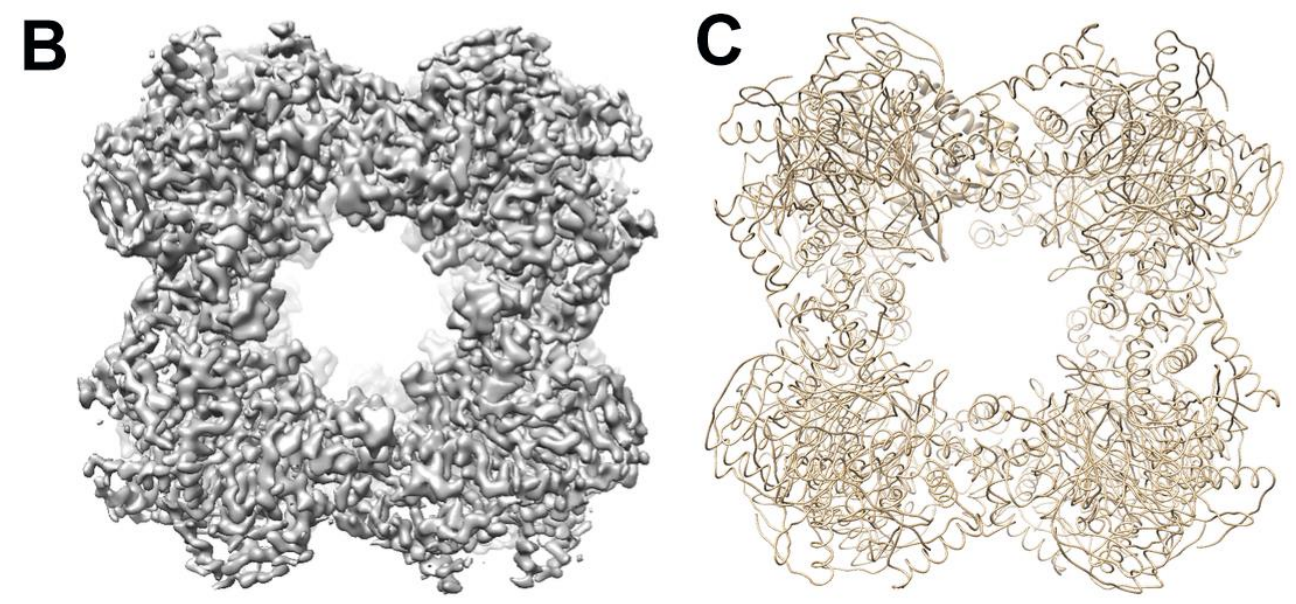

Figure 1. Structure of dihydrolipoamide succinyl transferase component of 2-oxoglutarate dehydrogenase complex. (A) Domain structure. The domains are highlighted as follows: light gray - lypoil-binding domain; dark gray - E3-binding domain; underlined - catalytic domain. Identified by tandem mass-speck peptides are indicated by bold. (B) Cryo-EM structure of 24-meric enzyme; (C) atomic de novo model 


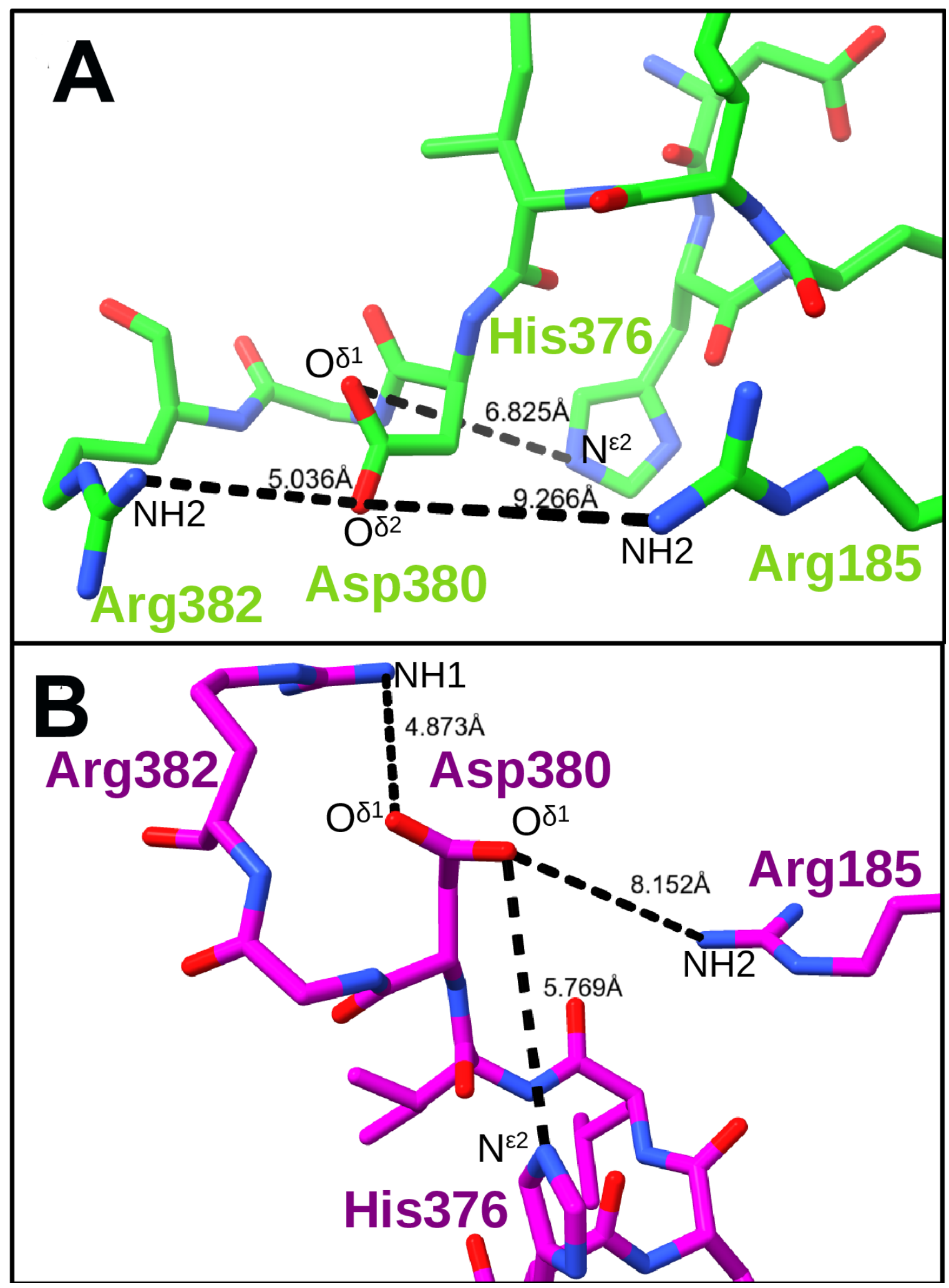

Figure 2. Comparison of the cryo-EM and crystal structures of an active center of dihydrolipoamid succinyltransferase catalytic domain. (A) The active site in the structure, obtained by X-ray crystallography (6prb) [5]; (B) The active site in the structure, obtained by cryo-EM. 


\section{References}

[1] Scheres, S.H.W. Journal of structural biology 180.3 (2012): 519-530.

[2] Punjani, A., et al. Nature methods 14.3 (2017): 290.

[3] Emsley, P., et al. "Features and development of Coot." Acta Crystallographica Section D: Biological Crystallography 66.4 (2010): 486-501.

[4] Knapp, J.E., et al. Journal of molecular biology 280.4 (1998): 655-668.

[5] Andi, B., et al. Acta Crystallographica Section F: Structural Biology Communications 75.9 (2019): 616-624.

[6] Cryo-EM sample preparation was performed using the "3D-EMC" instrument cluster of Moscow State University (supported by the Ministry of science and higher education of Russian Federation, unique identifier RFMEFI61919X0014). 